Christian Bucher

\title{
A family of triangular and tetrahedral elements with rotational degrees of freedom
}

This paper is dedicated to the memory of Franz Ziegler

Received: 30 March 2017 / Revised: 26 July 2017 / Published online: 21 November 2017

(C) The Author(s) 2017. This article is an open access publication

\begin{abstract}
A purely kinematic approach to the formulation of plane stress/strain triangular elements with three nodes as well as tetrahedral elements with four nodes including rotational degrees of freedom is presented. The class of elements is shown to perform comparably well in several well-established test cases. Essentially, the displacement interpolation functions are cubic allowing for more flexibility in the displacement-based formulation. The in-plane triangle can be augmented by a plate bending element to form an efficient triangular shell element.
\end{abstract}

\section{Introduction}

Triangular and tetrahedral elements are useful since it is very easy to generate such meshes for fairly arbitrary geometries automatically. It can, however, be observed that simplicial (i.e., triangular and tetrahedral) elements when using an isoparametric formulation for the displacements fields typically are too stiff. One well-known example for this behavior is shear locking of the constant stress triangle (CST) element [3]. In order to circumvent these problems, several strategies have been proposed, such as the assumed strain concept (e.g., [4, $10,14]$ ) or the introduction of additional degrees of freedom, e.g., drilling DOF's $[1,7,12,15,18]$. A very general overview is given, e.g., by [9].

This paper aims at laying out a methodology to derive simplicial element formulations based on nodal translations and nodal rotations. Focus is put on making sure that the elements derived are sufficiently flexible such that reasonable accuracy can be achieved with moderate mesh resolution. This is of special importance when the structural analysis needs to be repeated multiple times which is typically the case when structural optimization is carried out, or when stochastic analysis is performed [5], or when both are carried out simultaneously. In such cases, mesh resolution is a critical factor together with acceptable accuracy.

\section{Method of analysis}

The concept of rotation is not necessarily well defined in a continuum. One possibility of defining a rotation field within a continuum is to utilize the mathematical concept of a rotation vector. For a given three-dimensional vector field $\mathbf{u}=\left[\begin{array}{lll}u & v & w\end{array}\right]^{\mathrm{T}}$, the rotation can be defined in terms of the rotor (or curl) vector:

$$
\mathbf{r}=\operatorname{curl} \mathbf{u}=\nabla \times \mathbf{u}=\left[\begin{array}{c}
w_{y}-v_{z} \\
u_{z}-w_{x} \\
v_{x}-u_{y}
\end{array}\right]
$$

C. Bucher $(\varangle)$

Technische Universität Wien, Karlsplatz 13, 1040 Vienna, Austria

E-mail: christian.bucher@tuwien.ac.at 


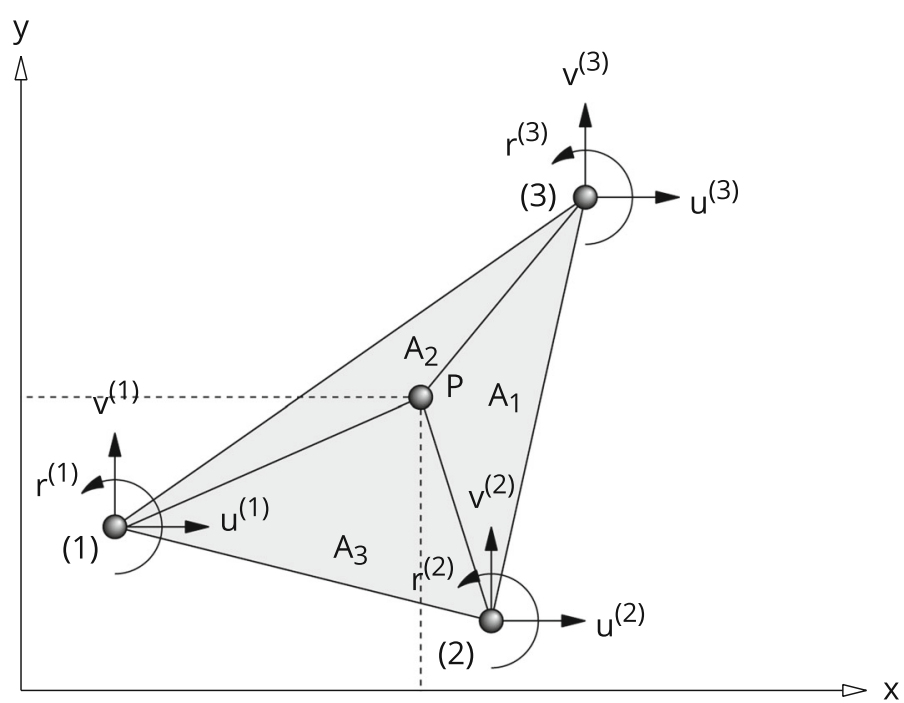

Fig. 1 Three-noded finite element QTRI3

Here the indices indicate partial derivatives of the vector components with respect to the spatial coordinates $x$, $y$, and $z$. For the case of a 2D displacement field containing the components $u$ and $v$, obviously only the third component of the curl vector, i.e., $v_{x}-u_{y}$ remains.

An arbitrary point $P$ with coordinates $x$ and $y$ within a triangular element (cf. Fig. 1) can be uniquely identified in terms of triangular (area) coordinates $\lambda_{k}=\frac{A_{k}}{A}$. Here $A$ denotes the area of the triangle and $A_{k}$ the area of the sub-triangle located opposite to node $k$ (cf. Fig. 1). They can be computed according to

$$
\begin{aligned}
\lambda_{1}(x, y) & =\frac{x_{2} y_{3}-x y_{3}-x_{3} y_{2}+x y_{2}+x_{3} y-x_{2} y}{2 A}, \\
\lambda_{2}(x, y) & =\frac{-x_{1} y_{3}+x y_{3}+x_{3} y_{1}-x y_{1}-x_{3} y+x_{1} y}{2 A}, \\
\lambda_{3}(x, y) & =1-\lambda_{1}-\lambda_{2}, \\
2 A & =\left|x_{2}\left(y_{3}-y_{1}\right)+x_{1}\left(y_{2}-y_{3}\right)+x_{3}\left(y_{1}-y_{2}\right)\right| .
\end{aligned}
$$

Note that for each node $k$ only $\lambda_{k}$ is nonzero, the other $\lambda_{\ell}$ are zero. For the subsequent analysis, it is helpful to compute the partial derivatives of $\lambda_{k}$ with respect to the coordinates $x$ and $y$ :

$$
\begin{array}{ll}
\lambda_{1, x}=\frac{y_{2}-y_{3}}{2 A}, & \lambda_{1, y}=\frac{x_{3}-x_{2}}{2 A}, \\
\lambda_{2, x}=\frac{y_{3}-y_{1}}{2 A}, & \lambda_{2, y}=\frac{x_{1}-x_{3}}{2 A}, \\
\lambda_{3, x}=\frac{y_{1}-y_{2}}{2 A}, & \lambda_{3, y}=\frac{x_{2}-x_{1}}{2 A} .
\end{array}
$$

For the interpolation of the displacements due to the nodal rotations, the original Allman's triangle uses a quadratic function [1]. Later, a cubic interpolation has been proposed [2]. This cubic interpolation can be elegantly achieved by assuming the shape functions for the nodal rotations in the form of

$$
\begin{aligned}
& u_{r}^{(k)}(x, y)=-\left(y-y^{(k)}\right) \lambda_{k}^{2}, \\
& v_{r}^{(k)}(x, y)=\left(x-x^{(k)}\right) \lambda_{k}^{2} .
\end{aligned}
$$

These functions are cubic in the coordinates $x$ and $y$. Obviously, the displacements $u$ and $v$ introduced by the nodal rotations at all nodes are equal to zero. The rotations introduced by these shape functions are given by

$$
r_{r}^{(k)}(x, y)=\frac{1}{2}\left(\frac{\partial v_{r}^{(k)}}{\partial x}-\frac{\partial u_{r}^{(k)}}{\partial y}\right)=\left(x-x^{(k)}\right) \lambda_{k} \frac{\partial \lambda_{k}}{\partial x}+\left(y-y^{(k)}\right) \lambda_{k} \frac{\partial \lambda_{k}}{\partial y}+\lambda_{k}^{2} .
$$


At the nodes $k$, these rotations evaluate to unity while at the remaining nodes they are zero. Note that the nodal rotations $r^{(k)}$ are introduced as additional degrees of freedom which are to be interpreted as relative rotations superimposed on the displacement field and the average rotation generated by the nodal displacements $u^{(k)}$ and $v^{(k)}$. This notion of "hierarchical rotations" has been discussed in [1,2] and elaborated on in detail in [9]. Hence the nodal rotations are formally defined as

$$
r^{(k)}=r_{r}^{(k)}\left(x^{(k)}, y^{(k)}\right) \text {. }
$$

The shape functions for the displacements due to the nodal displacements are assumed to be linear (which corresponds to the constant strain triangle),

$$
\begin{aligned}
& u_{u}^{(k)}=\lambda_{k} ; \quad u_{v}^{(k)}=0, \\
& v_{u}^{(k)}=0 ; \quad v_{v}^{(k)}=\lambda_{k} .
\end{aligned}
$$

These functions have unit values at node $k$ and vanish at the other nodes. The rotation fields due to the displacements $u$ and $v$ at node $k$ are readily computed as

$$
\begin{aligned}
& r_{u^{(k)}}=\frac{1}{2}\left(\frac{\partial v_{u}^{(k)}}{\partial x}-\frac{\partial u_{u}^{(k)}}{\partial y}\right)=-\frac{1}{2} \frac{\partial \lambda_{k}}{\partial y}, \\
& r_{v^{(k)}}=\frac{1}{2}\left(\frac{\partial v_{v}^{(k)}}{\partial x}-\frac{\partial u_{v}^{(k)}}{\partial y}\right)=\frac{1}{2} \frac{\partial \lambda_{k}}{\partial x}
\end{aligned}
$$

which, as can be seen from Eq. (3), are constant in the element.

Combining the results as derived above, the displacement fields $u(x, y)$ and $v(x, y)$ due to nodal displacements $u^{(k)}$ and $v^{(k)}$ and nodal rotation $r^{(k)}$ can be expressed as

$$
\begin{aligned}
& u(x, y)=\sum_{k=1}^{3} \lambda_{k} u^{(k)}-\left(y-y^{(k)}\right) \lambda_{k}^{2} r^{(k)}, \\
& v(x, y)=\sum_{k=1}^{3} \lambda_{k} v^{(k)}+\left(x-x^{(k)}\right) \lambda_{k}^{2} r^{(k)} .
\end{aligned}
$$

This can be written in compact form,

$$
\left[\begin{array}{c}
u \\
v
\end{array}\right]=\mathbf{H}_{e} \mathbf{u}_{e} ; \mathbf{u}_{e}=\left[\begin{array}{lllll}
u^{1} & v^{1} r^{1} u^{2} v^{2} r^{2} u^{3} v^{3} r^{3}
\end{array}\right]^{\mathrm{T}},
$$

in which the elements of the matrix $\mathbf{H}_{e}$ can be easily recovered from Eq. (9).

If the element undergoes a rigid-body translation (i.e., $u^{(k)}=u_{0}, v^{(k)}=v_{0}$ and $r^{(k)}=0$ for all $k$ ), then one obtains

$$
\begin{aligned}
& u(x, y)=u_{0}\left(\lambda_{1}+\lambda_{2}+\lambda_{3}\right)=u_{0}=\text { const. } \\
& v(x, y)=v_{0}\left(\lambda_{1}+\lambda_{2}+\lambda_{3}\right)=v_{0}=\text { const. }
\end{aligned}
$$

and hence the element is strain free. For an imposed rigid-body rotation, all nodal rotations due to the translational degrees of freedom are the identical, and therefore the (hierarchical) rotations $r^{(k)}$ interpreted as deviations from the average element rotation are zero, and hence the element is strain free.

From Eq. (9), the standard linearized strains are readily derived as

$$
\begin{aligned}
& \varepsilon_{x x}=\frac{\partial u}{\partial x}=\sum_{k=1}^{3} \frac{\partial \lambda_{k}}{\partial x} u^{(k)}-2 \frac{\partial \lambda_{k}}{\partial x}\left(y-y^{(k)}\right) \lambda_{k} r^{(k)}, \\
& \varepsilon_{y y}=\frac{\partial v}{\partial y}=\sum_{k=1}^{3} \frac{\partial \lambda_{k}}{\partial y} v^{(k)}+2 \frac{\partial \lambda_{k}}{\partial y}\left(x-x^{(k)}\right) \lambda_{k} r^{(k)}, \\
& \gamma_{x y}=\frac{\partial u}{\partial y}+\frac{\partial v}{\partial x}=\sum_{k=1}^{3} \frac{\partial \lambda_{k}}{\partial y} u^{(k)}+\frac{\partial \lambda_{k}}{\partial x} v^{(k)}+\left[\frac{\partial \lambda_{k}}{\partial x}\left(x-x^{(k)}\right) \lambda_{k}-\frac{\partial \lambda_{k}}{\partial y}\left(y-y^{(k)}\right) \lambda_{k}\right] r^{(k)} .
\end{aligned}
$$




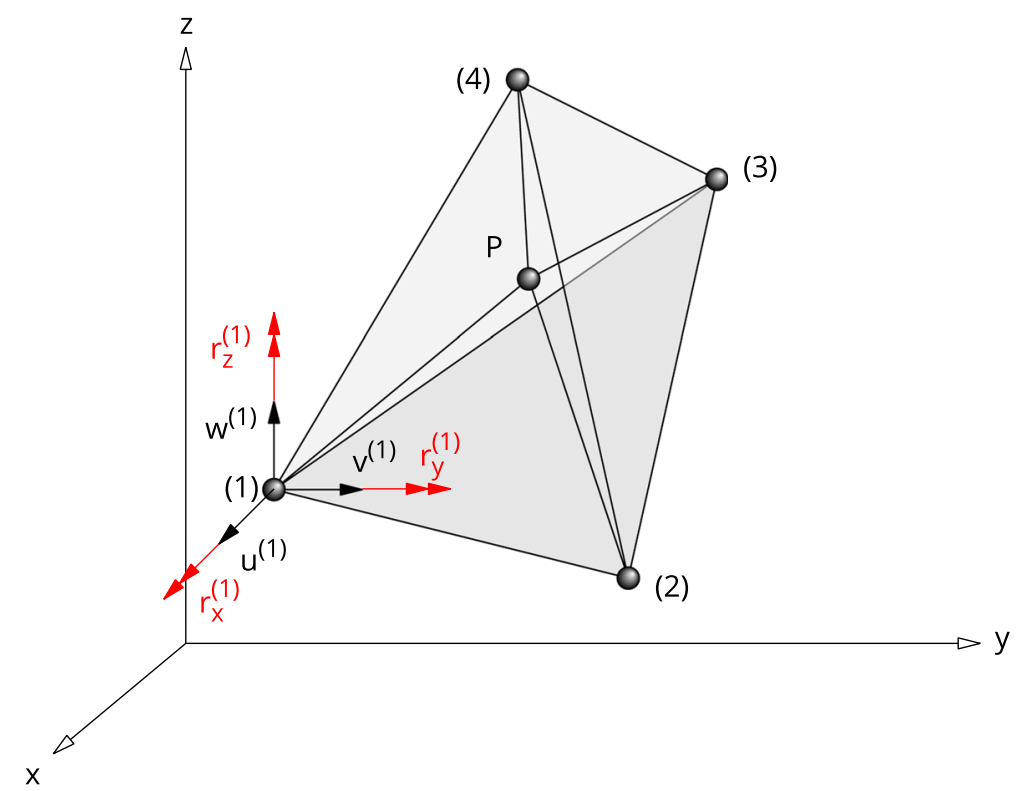

Fig. 2 Tetrahedral element QTET4 with rotational degrees of freedom

Again, this can be written more compactly as

$$
\left[\begin{array}{c}
\varepsilon_{x x} \\
\varepsilon_{y y} \\
\gamma_{x y}
\end{array}\right]=\mathbf{B}_{e} \mathbf{u}_{e}
$$

and the elements of the matrix $\mathbf{B}_{e}$ can be recovered from Eq. (12).

Based on these equations, expressions for the element stiffness and mass matrices $\mathbf{K}_{e}$ and $\mathbf{M}_{e}$ can be derived using standard finite element technology:

$$
\mathbf{K}_{e}=\int_{V_{e}} \mathbf{B}_{e}^{T} \mathbf{D} \mathbf{B}_{e} \mathrm{~d} V ; \mathbf{M}_{e}=\int_{V_{e}} \rho \mathbf{H}_{e}^{T} \mathbf{H}_{E} \mathrm{~d} V
$$

in which $\mathbf{D}$ denotes the material elasticity tensor in appropriate representation, $\rho$ is the material mass density, and $V_{e}$ denotes the element volume. The integration is carried out using under-integration with three integration points per element. This is sufficient to obtain a non-singular element stiffness matrix.

An analogous concept can be applied to develop tetrahedral elements with rotational degrees of freedom (cf. Fig. 2). The derivations now include the full rotation vector as defined in Eq. (1). The shape functions are then based on tetrahedral coordinates $\lambda_{i}, i=1 \ldots 4$. For a point with Cartesian coordinates $x, y, z$, the tetrahedral (barycentric) coordinates can be determined from

$$
\left[\begin{array}{l}
\lambda_{1} \\
\lambda_{2} \\
\lambda_{3}
\end{array}\right]=\left[\begin{array}{lll}
x_{1}-x_{4} & x_{2}-x_{4} & x_{3}-x_{4} \\
y_{1}-y_{4} & y_{2}-y_{4} & y_{3}-y_{4} \\
z_{1}-z_{z} & z_{2}-z_{4} & z_{3}-z_{4}
\end{array}\right]^{-1}\left[\begin{array}{l}
x-x_{4} \\
y-y_{4} \\
z-z_{4}
\end{array}\right] ; \lambda_{4}=1-\lambda_{1}-\lambda_{2}-\lambda_{3} .
$$

In the subsequent steps the derivation does not introduce any substantially new material as compared to the triangular element, therefore this is not re-iterated here. When carrying out the numerical analysis it turned out that a four-point integration (in analogy to the three-point integration for the QTRI3 element) does not result in a regular stiffness matrix. In order to achieve regularity, a fourth-order integration with 11 integration points is required. This, however, leads to an overly stiff element. Hence, a one-point under-integration has been applied. Although this yields singular element stiffness matrices, all numerical examples ran fine and did not trigger these singularities. If desired, the elements could easily be stabilized by regularizing the element matrices. It should be noted that in $[13,17]$ also tetrahedral elements with rotational degrees of freedom have been developed, but this derivation was based on mid-side nodes rather than nodal rotations only. 


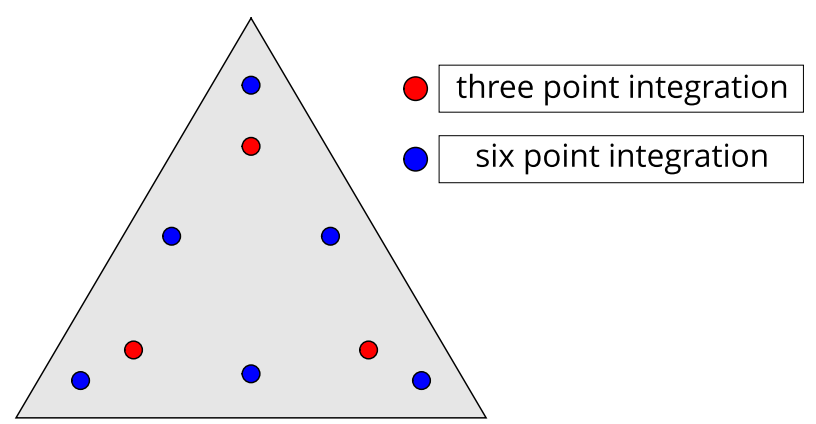

Fig. 3 Single triangular element

Table 1 Eigenvalues of the stiffness matrix for different integration schemes

\begin{tabular}{llc}
\hline Eigenvalue & Using 6 points & Using 3 points \\
\hline 1 & 0.0000 & 0.0000 \\
2 & 0.0000 & 0.0000 \\
3 & 0.0000 & 0.0000 \\
4 & 0.0069 & 0.0019 \\
5 & 0.0069 & 0.0019 \\
7 & 0.0770 & 0.0321 \\
8 & 0.8660 & 0.8660 \\
9 & 0.9024 & 0.9022 \\
\hline
\end{tabular}

\section{Numerical examples}

This Section treats two commonly used test examples, i.e., a cantilever beam under parabolic shear load and a trapezoidal console with uniformly distributed transverse end load (Cook's panel). Both structures are modeled on one hand by triangular elements (QTRI3 and others for comparison) and on the other hand by four-noded tetrahedral elements (QTET4 and others for comparison). The displacements in a characteristic point are computed for different mesh sizes and compared to each other. One final example computes the natural frequencies and mode shapes of a simply supported plate for which the analytical solution is known. The elements have been implemented into the open-source software package slangTNG [6].

\subsection{Stiffness properties of the triangular element}

In order to investigate the numerical properties of the QTRI3 element, consider the special case of an equilateral triangle as shown in Fig. 3.

For unit length, unit thickness, unit modulus of elasticity, and zero Poisson's ratio, the element stiffness matrix is computed and analyzed with respect to its eigenvalues. The nine eigenvalues are given in Table 1 for a fully integrated stiffness matrix (using six integration points) and an under-integrated stiffness matrix (using three integration points). The locations of the integration points are chosen based on [8].

Both variants contain the correct number of zero eigenvalues corresponding to two rigid-body translations and one rigid-body rotation in the plane of the triangle.

\subsection{Cook's panel using triangular elements}

This well-known structure as shown in Fig. 4 is analyzed as a first test for the QTRI3 element. More refined meshes are shown in Fig. 5. The material parameters are $E=1, v=1 / 3$. The thickness of the panel is 1 . The structure is loaded by a uniformly distributed load of total magnitude 1 as shown in Fig. 4. Plane stress conditions are assumed. The displacement of node $C$ is plotted as a function of the number $m$ of subdivisions along the edges as shown in Fig. 6. Figure 4 contains results using three different triangular element formulations and different mesh resolutions. The curve labeled as QTRI3 is the solution using the element proposed in this 


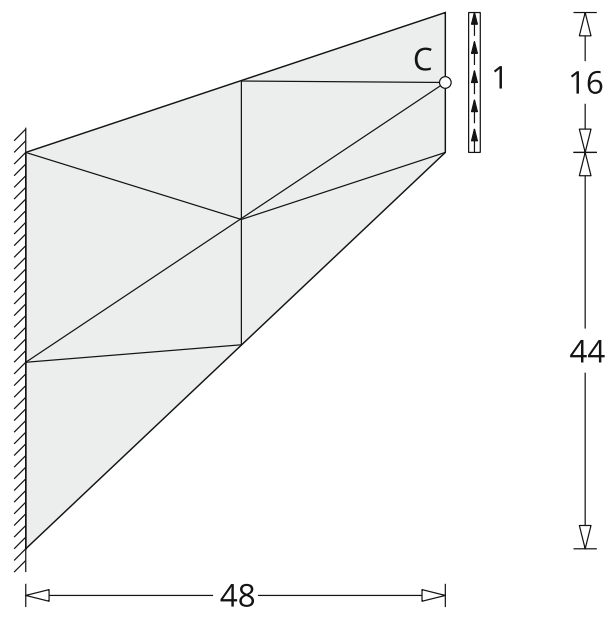

Fig. 4 Cook's panel and triangulation with $2 \times 2$ mesh
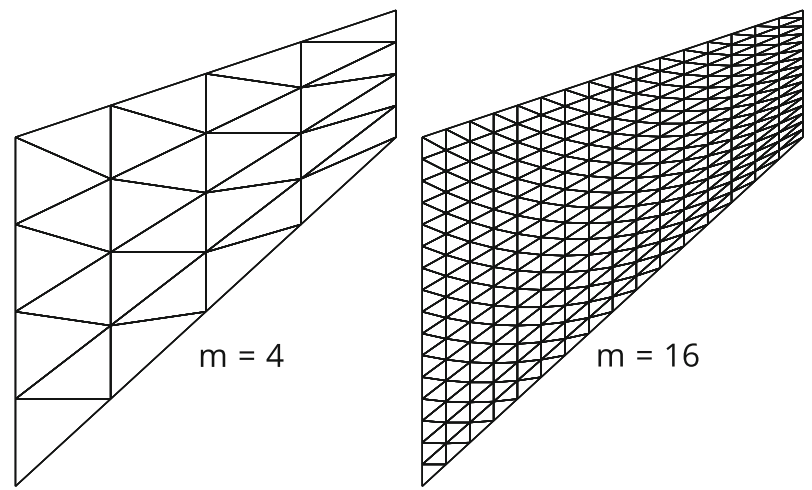

Fig. 5 Refined trinagular meshes for Cook's panel

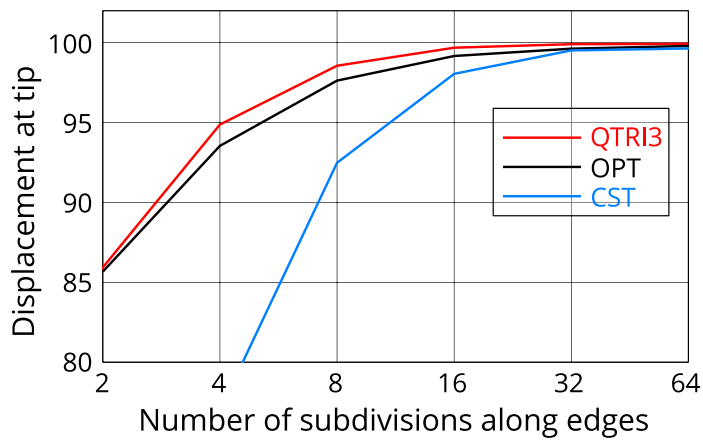

Fig. 6 Convergence of displacement for Cook's panel

paper. Label OPT indicates the solution presented in [9]. Both solutions are very close to each other. Solution CST is based on the constant strain triangle and clearly produces a structural behavior which is too stiff. All three solutions converge to the same result as the mesh resolution increases.

\subsection{Cantilever beam using triangular elements}

This example is a linear elastic beam as shown in Fig. 7. The rectangular area is triangulated using different mesh resolutions as shown in Fig. 8. Material parameters are $E=30000, v=1 / 4$. The thickness of the beam is assumed to be 1 . The total vertical load is 40 , which is distributed parabolically over the nodes at the free 


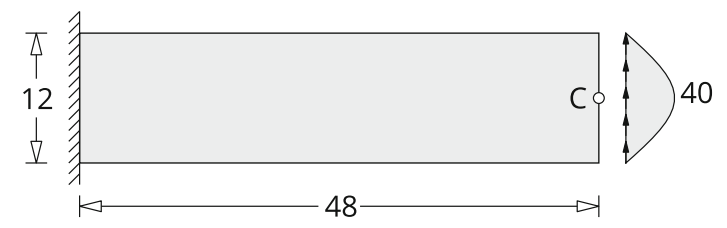

Fig. 7 Cantilever beam
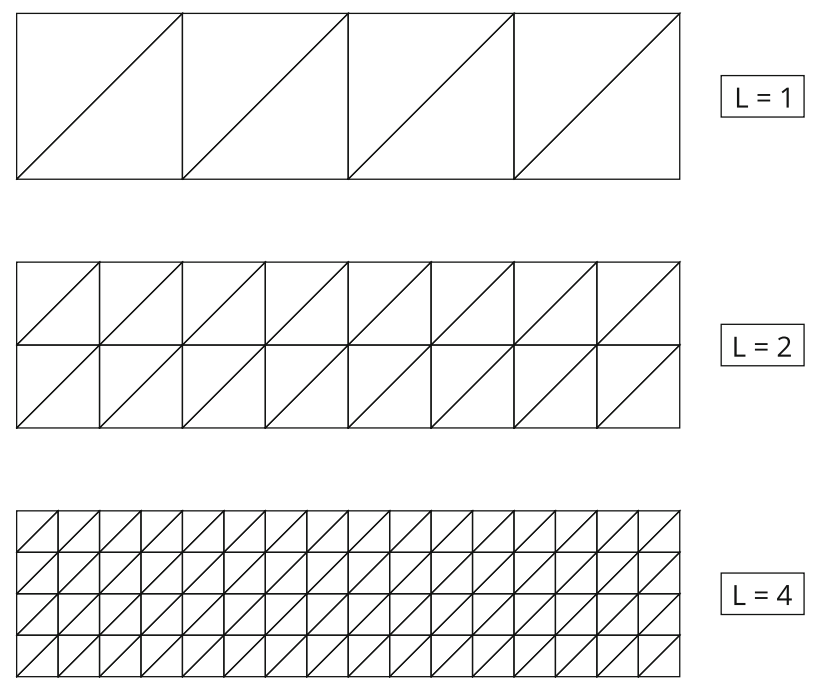

$\mathrm{L}=4$

Fig. 8 Triangular meshes for cantilever beam

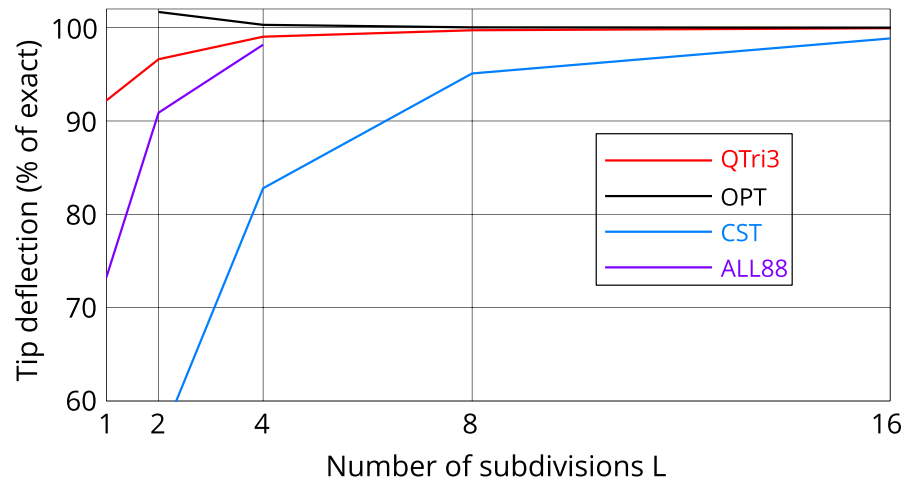

Fig. 9 Convergence of displacement for cantilever beam (triangular meshes)

end of the cantilever. Plane stress conditions are assumed. The results from the element QTRI3 as developed in this paper are again compared to those using the optimal element [9] and the constant strain triangle. In this example, it can be observed that the QTRI3 element converges quickly from below whereas the OPT element converges from above. The error from the OPT is slightly smaller than the error from the QTRI3 element. In this Figure, the results using Allman's 1988 triangle [2] are also included with the label ALL88. It can be seen that this element clearly behaves stiffer than the proposed QTRI3 element (Fig. 9). Again, the constant strain triangle is overly stiff. It should be noted that the displacement of the point $C(0.35601$ as given by [9]) is taken as reference (i.e., as 100\%). This solution is slightly larger than the analytical solution of 0.35533 as reported in [9]. 

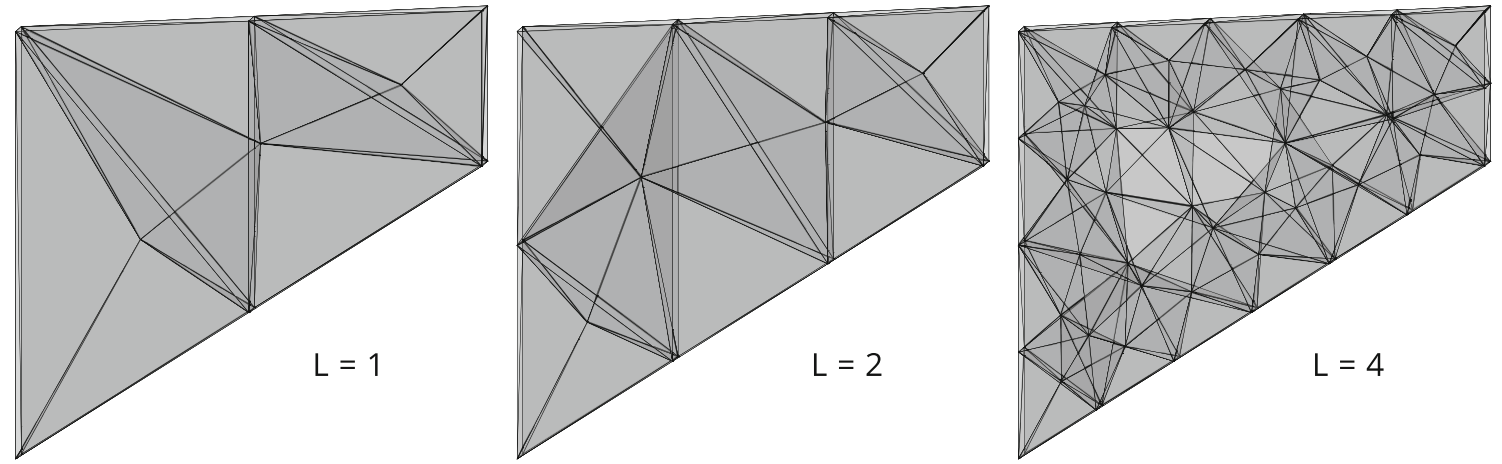

Fig. 10 Tetrahedral meshes for Cook's panel

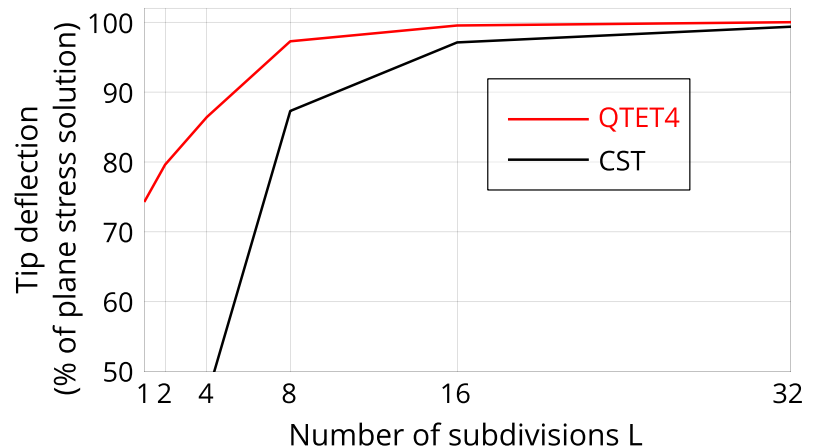

Fig. 11 Convergence of displacement for Cook's panel (tetrahedral meshes)

\subsection{Cook's panel using tetrahedral elements}

For this example, the panel as analyzed above is discretized using four-point tetrahedral elements. The same geometry and material parameters as above are used. Different tetrahedral meshes were generated using the open-source software tool gmsh [11]. Some of these meshes are shown in Fig. 10. The average vertical displacements of all nodes located on the loaded surface are taken as representative for the displacement and shown in Fig. 11 for different mesh resolutions. Results using the QTET4 element are compared to those obtained using the constant strain tetrahedron (CST) element. It can be seen that the QTET4 element exhibits significantly better performance.

\subsection{Cantilever beam using tetrahedral elements}

In this example, the beam as analyzed above is discretized using four-point tetrahedral elements. The same geometric data and material parameters are used as for the analysis with triangular elements. Different tetrahedral meshes were generated, some of which are shown in Fig. 12. Also in this example the average vertical displacements of all nodes located on the loaded surface are taken as representative for the displacement and shown in Fig. 13 for different mesh resolutions. Results using the QTET4 element are compared to those obtained using the constant strain tetrahedron (CST) element. The performance of the QTET4 element is clearly superior.

\subsection{Thick plate}

A square plate with side length 10 is considered. It is simply supported on all four lower edges. Horizontal movement is allowed in the supports such that there is no tension due to the overall bending of the plate. The plate thickness is 1 , so there is some shear contribution present when applying plate bending theory. The material data are $E=200 \times 10^{9}, v=0.3, \rho=8000$. For the numerical analysis the plate is modeled by QTET4 elements. Three meshes with different mesh resolutions are shown in Fig. 14. The exact fundamental frequency based on Mindlin plate theory as taken from [16] is $f_{0}=45.44291 / \mathrm{s}$. For different mesh resolutions, 

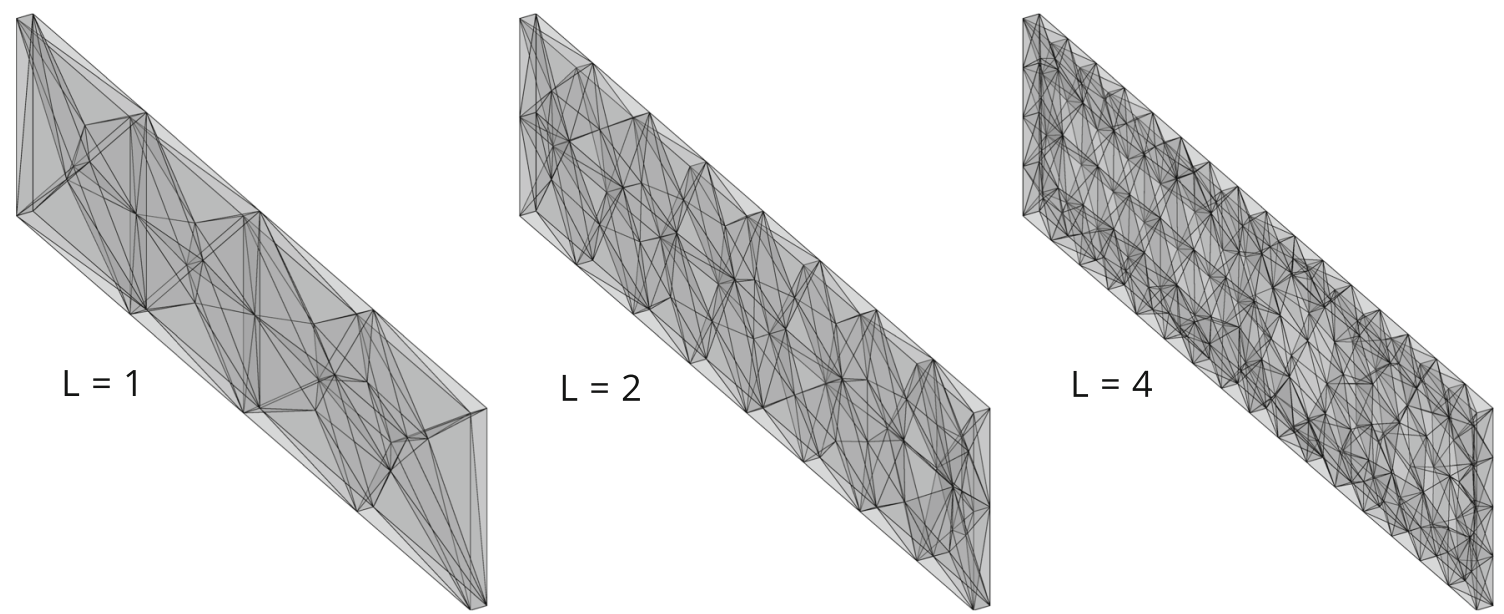

Fig. 12 Tetrahedrals meshes for cantilever beam

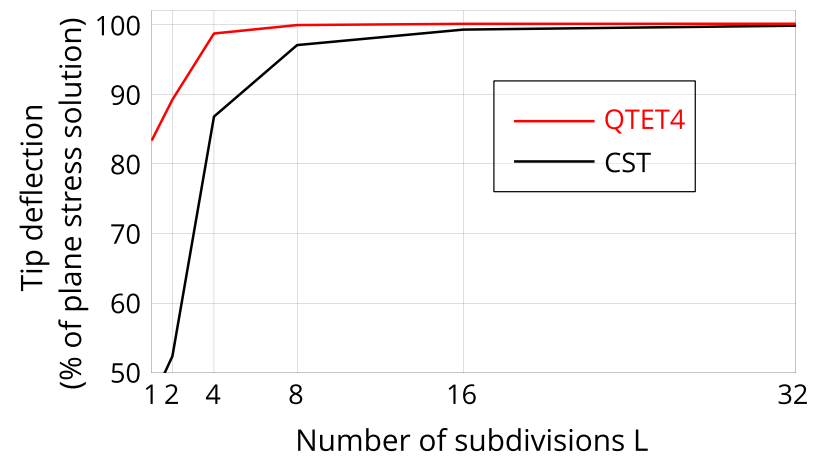

Fig. 13 Convergence of displacement for cantilever beam (tetrahedral elements)
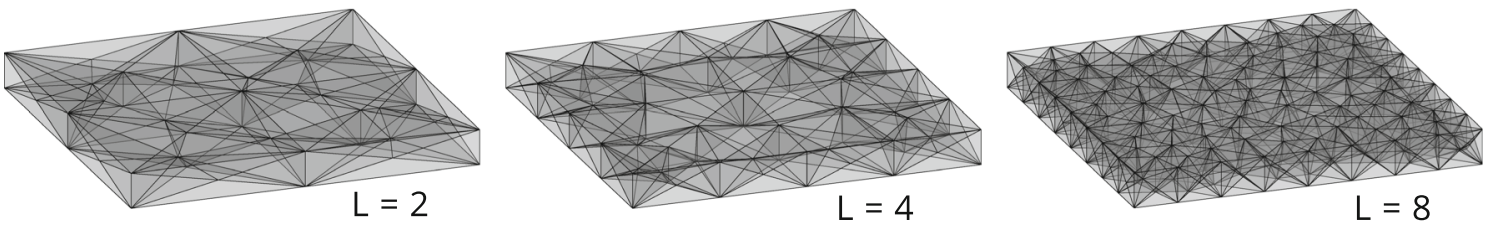

Fig. 14 Tetrahedral meshes for simply supported plate

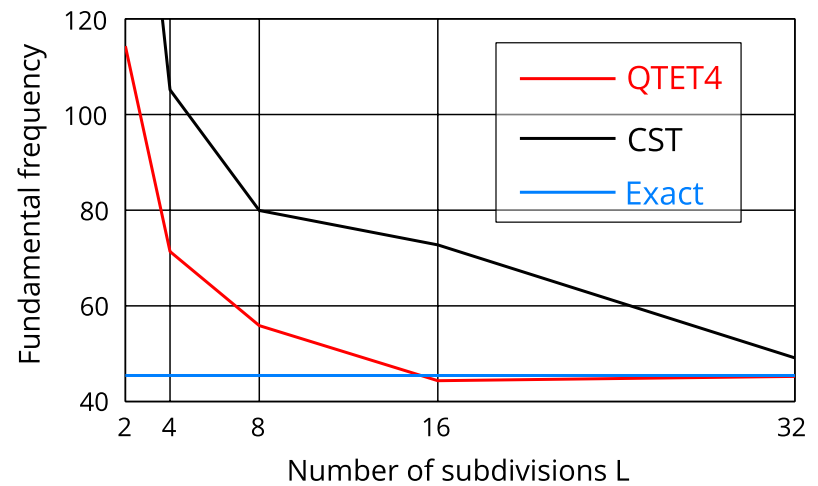

Fig. 15 Convergence of natural frequency for thick simply supported plate (tetrahedral elements)

the numerical results for the fundamental frequency are shown in Fig. 15. As in the previous examples, the proposed QTET4 element gives substantially better results when compared to the CST element. 


\section{Concluding remarks}

A family of simplicial elements with rotational degrees of freedom has been proposed, and its applicability was demonstrated using several classical test examples. It appears the both the triangular version (QTRI3) and the tetrahedral version (QTET4) perform reasonably well concerning the overall stiffness. This study did not include any results concerning stress recovery, which will be part of a future study on this element family.

Another point to be further clarified is under which circumstances the under-integration of the tetrahedral element might lead to numerical problems and what type of stabilization strategy might be most effective. Also, it remains to be seen how the performance of this element family develops in the presence of geometrical and material nonlinearities.

Acknowledgements Open access funding provided by Austrian Science Fund (FWF). This research was carried out within the Vienna Doctoral Programme on Water Resource Systems supported by the Austrian Science Funds (FWF) under contract W1219-N22.

Open Access This article is distributed under the terms of the Creative Commons Attribution 4.0 International License (http:// creativecommons.org/licenses/by/4.0/), which permits unrestricted use, distribution, and reproduction in any medium, provided you give appropriate credit to the original author(s) and the source, provide a link to the Creative Commons license, and indicate if changes were made.

\section{References}

1. Allman, D.J.: A compatible triangular element including vertex rotations for plane strain analysis. Comput. Struct. 19, 1-8 (1984)

2. Allman, D.J.: Evaluation of the constant strain triangle with drilling rotations. Int. J. Numer. Methods Eng. 26, 2645-2655 (1988)

3. Bathe, K.J.: Finite Element Procedures. Prentice Hall, Englewood Cliffs (1996)

4. Bletzinger, K.U., Bischoff, M., Ramm, E.: A unified approach for shear-locking-free triangular and rectangular shell finite elements. Comput. Struct. 75, 321-334 (2000)

5. Bucher, C.: Computational Analysis of Randomness in Structural Mechanics. Structures and Infrastructures Book Series, vol. 3. Taylor \& Francis, London (2009)

6. Bucher, C., Wolff, S.: slangTNG-software for stochastic structural analysis made easy. Meccanica dei Materiali e delle Strutture 3(4), 10-17 (2012)

7. Cook, R.D.: On the Allman triangle and related quadrilateral element. Comput. Struct. 22, 1065-1067 (1986)

8. Cowper, G.R.: Gaussian quadrature formulas for triangles. Int. J. Numer. Methods Eng. 7, 405-408 (1973)

9. Felippa, C.A.: A study of optimal membrane triangles with drilling freedoms. Comput. Methods Appl. Mech. Eng. 192, 2125-2168 (2003)

10. Flores, F.G.: A two-dimensional linear assumed strain triangular element for finite deformation analysis. J. Appl. Mech. 73, 970-976 (2006)

11. Geuzaine, C., Remacle, J.F.: Gmsh. http://geuz.org/gmsh/doc/texinfo/gmsh.html

12. Huang, M., Zhao, Z., Shen, C.: An effective planar triangular element with drilling rotation. Finite Elem. Anal. Des. 48, 1031-1036 (2010)

13. Pawlak, T.K., Yunus, S.M., Cook, R.C.: Solid elements with rotational degrees of freedom: part II-tetrahedron element. Int. J. Numer. Methods Eng. 31, 593-610 (1991)

14. Simo, J., Rifa, M.: A class of mixed assumed strain methods and the method of incompatible modes. Int. J. Numer. Methods Eng. 29, 1595-1683 (1990)

15. Tian, R., Yagawa, G.: Allman's triangle, rotational DOF and partition of unity. Int. J. Numer. Methods Eng. 69, 837-858 (2007)

16. Wang, C.M.: Natural frequencies formula for simply supported Mindlin plates. J. Vib. Acoust. 116, 536-540 (1994)

17. Yunus, S.M., Pawlak, T.K., Cook, R.D.: Solid elements with rotational degrees of freedom: part I-hexahedron element. Int. J. Numer. Methods Eng. 31, 573-592 (1991)

18. Yunus, S.M., Saigal, S., Cook, R.D.: On improved hybrid finite elements with rotational degrees of freedom. Int. J. Numer. Methods Eng. 28, 785-800 (1989) 